\title{
PREFAZIONE DEL TRADUTTORE
}

Nel presentare ai lettori di questo Trattato la parte dedicata alle malattie del sistema nervoso centrale, desidero richiamare ancora una volta l'attenzione sulla utilità di esso per gli studenti di medicina e per gli specializzandi di Neurologia e Psichiatria. I nomi dei collaboratori (Staemmler, Peters, Zülch, ecc.) sono ben noti agli studiosi della patologia del sistema nervoso e danno il più ampio affidamento della serietà e della completezza di ogni singolo capitolo.

Un particolare ringraziamento, per quanto attiene alla traduzione italiana, va rivolto ai proff. Amico Bignami, Battistina Masini e Luigi Matturri, che hanno collaborato con competenza e con spirito di sacrificio all'attenta revisione e correzione delle bozze.

La Casa Editrice Vallardi ha curato con sensibilità e comprensione sia la veste tipografica sia le numerose illustrazioni, molte delle quali a colori provengono da materiale dell'Istituto di Anatomia e Istologia Patologica dell'Università di Milano, fotografato dal sig. Osvaldo Ciarrocca, al quale rivolgo, anche a nome dei collaboratori, il più vivo ringraziamento.

\section{Alfonso GIordano}


